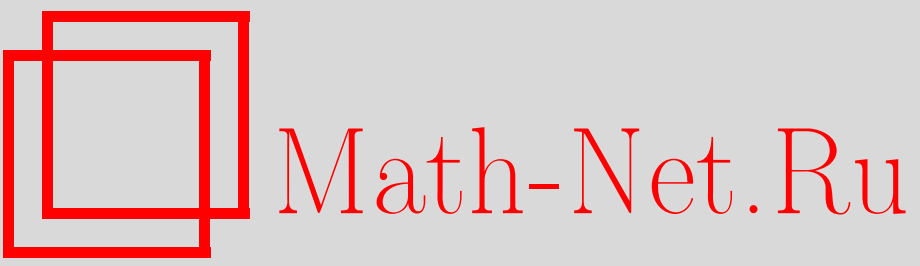

В. П. Спиридонов, Модулярность и полная эллиптичность некоторых многократных рядов гипергеометрического типа, ТMФ, 2003, том 135, номер 3, 462477

DOI: https://doi.org/10.4213/tmf203

Использование Общероссийского математического портала Math-Net.Ru подразумевает, что вы прочитали и согласны с пользовательским соглашением http://www.mathnet.ru/rus/agreement

Параметры загрузки:

IP : 54.198 .55 .26

26 апреля 2023 г., $17: 57: 06$ 
(C) 2003 г.

\section{МОДУЛЯРНОСТЬ И ПОЛНАЯ ЭЛЛИПТИЧНОСТЬ НЕКОТОРЫХ МНОГОКРАТНЫХ РЯДОВ ГИПЕРГЕОМЕТРИЧЕСКОГО ТИПА}

Приводятся новые аргументы в пользу справедливости гипотезы о том, что любой полностью эллиптический гипергеометрический ряд модулярен. Кратко обсуждается обобщение таких рядов на римановы поверхности произвольного рода.

Ключевые слова: тета-функции, эллиптические гипергеометрические ряды, модулярная группа, формулы суммирования.

Следуюшее общее определение эллиптических гипергеометрических функций было дано в работе [1].

ОПРЕДЕЛЕНИЕ. Формальные (многократные) ряды

$$
\sum_{\lambda_{1}, \ldots, \lambda_{n}=0}^{\infty} c\left(\lambda_{1}, \ldots, \lambda_{n}\right) \quad \text { или } \sum_{0 \leqslant \lambda_{1} \leqslant \cdots \leqslant \lambda_{n} \leqslant \infty} c\left(\lambda_{1}, \ldots, \lambda_{n}\right)
$$

определяют әллиптические гипергеометрические функиии, если:

1) коэффициенты $c(\boldsymbol{\lambda})$ симметричны относительно действия группы перестановок $\mathcal{S}_{n}$ на индексы суммирования $\lambda_{1}, \ldots, \lambda_{n}$ и свободные параметры, входящие в $c(\boldsymbol{\lambda})$;

2) для любых $\ell=1, \ldots, n$ отношения

$$
h_{\ell}(\boldsymbol{\lambda})=\frac{c\left(\lambda_{1}, \ldots, \lambda_{\ell}+1, \ldots, \lambda_{n}\right)}{c\left(\lambda_{1}, \ldots, \lambda_{\ell}, \ldots, \lambda_{n}\right)}
$$

являются эллиптическими функциями индексов $\lambda_{\ell}, \ell=1, \ldots, n$, рассматриваемых как комплексные переменные.

Удобно принять начальное условие $c(0, \ldots, 0)=1$, при котором коэффициенты $c\left(\lambda_{1}, \ldots, \lambda_{n}\right), \lambda_{j} \in \mathbb{N}$, однозначно определяются функциями $h_{\ell}(\boldsymbol{\lambda})$ после решения $n$ рекуррентных соотношений первого порядка (2).

${ }^{*}$ Институт математики им. Макса Планка, Бонн, Германия; Лаборатория теоретической фиизики им. Н.Н. Боголюбова, Объединенный институт ядерных исследований, Дубна, Московская обл., Россия. E-mail: spiridon@thsun1.jinr.ru 
При $n=1$ отправной точкой служит обшая эллиптическая функция порядка $r+1$, которая может быть факторизована следуюшим образом [2]:

$$
\begin{gathered}
h(\lambda)=z \frac{\theta_{1}\left(u_{0}+\lambda, \ldots, u_{r}+\lambda ; \sigma, \tau\right)}{\theta_{1}\left(v_{0}+\lambda, \ldots, v_{r}+\lambda ; \sigma, \tau\right)}=z \frac{\theta\left(t_{0} q^{\lambda}, \ldots, t_{r} q^{\lambda} ; p\right)}{\theta\left(w_{0} q^{\lambda}, \ldots, w_{r} q^{\lambda} ; p\right)}, \\
\theta_{1}\left(u_{0}, \ldots, u_{k} ; \sigma, \tau\right)=\prod_{i=0}^{k} \theta_{1}\left(u_{i} ; \sigma, \tau\right), \quad \theta\left(t_{0}, \ldots, t_{k} ; p\right)=\prod_{i=0}^{k} \theta\left(t_{i} ; p\right),
\end{gathered}
$$

где $p=e^{2 \pi i \tau}, \operatorname{Im} \tau>0, q=e^{2 \pi i \sigma}$ и функция

$$
\begin{aligned}
\theta_{1}(u ; \sigma, \tau) & =-i \sum_{n=-\infty}^{\infty}(-1)^{n} p^{(2 n+1)^{2} / 8} q^{(n+1 / 2) u}= \\
& =p^{1 / 8} i q^{-u / 2}(p ; p)_{\infty} \theta\left(q^{u} ; p\right), \quad u \in \mathbb{C},
\end{aligned}
$$

является одной из стандартных тета-функций Якоби. Функция $\theta(z ; p)$ представляется бесконечным произведением вида

$$
\theta(z ; p)=(z ; p)_{\infty}\left(p z^{-1} ; p\right)_{\infty}, \quad(a ; p)_{\infty}=\prod_{n=0}^{\infty}\left(1-a p^{n}\right)
$$

и обладает следующими свойствами:

$$
\theta(p z ; p)=\theta\left(z^{-1} ; p\right)=-z^{-1} \theta(z ; p)
$$

Иногда для краткости удобно опускать в обозначениях для тета-функций или эллиптических сдвинутых факториалов базовые $(q, p)$ или модулярные $(\sigma, \tau)$ параметры. Мы будем пользоваться также обозначением для эллиптических чисел [3]

$$
[u ; \sigma, \tau] \equiv \theta_{1}(u ; \sigma, \tau) \quad \text { или } \quad[u] \equiv \theta_{1}(u) .
$$

Параметры $u_{i}, v_{i}, t_{i}, w_{i}$ в $(3)$ связаны друг с другом соотношениями $t_{i}=q^{u_{i}}, w_{i}=q^{v_{i}}$ и удовлетворяют ограничениям

$$
\sum_{i=0}^{r}\left(u_{i}-v_{i}\right)=0 \quad \text { или } \quad \prod_{i=0}^{r} t_{i}=\prod_{i=0}^{r} w_{i},
$$

которые гарантируют, что мероморфная функция $h(\lambda)$ дважды периодическая:

$$
h\left(\lambda+\sigma^{-1}\right)=h(\lambda), \quad h\left(\lambda+\tau \sigma^{-1}\right)=h(\lambda) .
$$

Напомним, что функция $[u]$ является целой, нечетной $([-u]=-[u])$ и дважды квазипериодической:

$$
\left[u+\sigma^{-1}\right]=-[u], \quad\left[u+\tau \sigma^{-1}\right]=-e^{-\pi i \tau-2 \pi i \sigma u}[u]
$$


Модулярная $P S L(2, \mathbb{Z})$ группа,

$$
\tau \rightarrow \frac{a \tau+b}{c \tau+d}, \quad \sigma \rightarrow \frac{\sigma}{c \tau+d}
$$

где $a, b, c, d \in \mathbb{Z}$ и $a d-b c=1$, генерируется двумя преобразованиями: $\tau \rightarrow \tau+1, \sigma \rightarrow \sigma$ и $\tau \rightarrow-\tau^{-1}, \sigma \rightarrow \sigma \tau^{-1}$. В этих двух случаях мы имеем

$$
\begin{aligned}
{[u ; \sigma, \tau+1] } & =e^{\pi i / 4}[u ; \sigma, \tau], \\
{[u ; \sigma / \tau,-1 / \tau] } & =i(-i \tau)^{1 / 2} e^{\pi i \sigma^{2} u^{2} / \tau}[u ; \sigma, \tau]
\end{aligned}
$$

и знак корня $(-i \tau)^{1 / 2}$ фиксируется условием положительности его реальной части.

Поместим один из полюсов функции $h(\lambda)(3)$ в точку $\lambda=-1$, положив $v_{0}=1$ или $w_{0}=q$ (что является обшепринятой нормировкой). Результируюшая функция $h(\lambda)$ порождает эллиптический гипергеометрический ряд одной переменной, который мы представим в мультипликативной системе обозначений:

$$
{ }_{r+1} E_{r}\left(\begin{array}{c}
t_{0}, \ldots, t_{r} \\
w_{1}, \ldots, w_{r}
\end{array} ; q, p ; z\right)=\sum_{\lambda=0}^{\infty} \frac{\theta\left(t_{0}, t_{1}, \ldots, t_{r} ; p ; q\right)_{\lambda}}{\theta\left(q, w_{1}, \ldots, w_{r} ; p ; q\right)_{\lambda}} z^{\lambda}
$$

где эллиптические сдвинутые факториалы определены следующим образом:

$$
\theta\left(t_{0}, \ldots, t_{k} ; p ; q\right)_{\lambda} \equiv \prod_{m=0}^{k} \prod_{j=0}^{\lambda-1} \theta\left(t_{m} q^{j} ; p\right)
$$

В аддитивной форме эллиптические сдвинутые факториалы имеют вид

$$
\left[u_{0}, \ldots, u_{k}\right]_{\lambda} \equiv \prod_{m=0}^{k} \prod_{j=0}^{\lambda-1}\left[u_{m}+j ; \sigma, \tau\right]
$$

Ряды (11) могут быть определены и без условия эллиптичности (6). Поэтому удобно называть общие ряды гипергеометрического типа, построенные из тета-функций Якоби, тета-гипергеометрическими рядами. В этом случае равенства (6) называются условием балансировки.

Можно расширить определение (1), (2), заменив двойную периодичность на квазипериодичность (7), характерную для тета-функций Якоби. В результате получаются тета-функциональные аналоги обычных и базисных обобшенных гипергеометрических рядов ${ }_{s} F_{r}$ и $_{s} \Phi_{r}$, не содержаших ограничений на параметры типа (6) (подробнее см. [1]).

Тета-гипергеометрические ряды (11) называются полностью әллиптическими, если функция $h(\lambda)(3)$ эллиптична не только по переменной $\lambda$, но также и по всем свободным параметрам среди $u_{i}, v_{i}$. Как показано в [1], требование полной эллиптичности приводит к дополнительным ограничениям на параметры

$$
u_{0}+1=u_{1}+v_{1}=\cdots=u_{r}+v_{r} \text { или } q t_{0}=t_{1} w_{1}=\cdots=t_{r} w_{r}
$$


которые хорошо известны как условия вполне уравновешенности для обычных и базисных гипергеометрических рядов [4]. Это дает некоторое объяснение происхождения самого этого понятия.

В эллиптическом случае условие совершенной уравновешенности отличается от соответствующих условий для обычных и базисных гипергеометрических рядов [4]. А именно, оно требует, чтобы в дополнение к условиям (12) четыре параметра были помещены в четыре точки второго порядка эллиптической функции $h(\lambda)$ вокруг некоторой выделенной точки:

$$
\begin{aligned}
u_{r-3} & =\frac{1}{2} u_{0}+1, & u_{r-2} & =\frac{1}{2} u_{0}+1-\frac{1}{2 \sigma}, \\
u_{r-1} & =\frac{1}{2} u_{0}+1-\frac{\tau}{2 \sigma}, & u_{r} & =\frac{1}{2} u_{0}+1+\frac{1+\tau}{2 \sigma},
\end{aligned}
$$

или в мультипликативной форме

$$
t_{r-3}=t_{0}^{1 / 2} q, \quad t_{r-2}=-t_{0}^{1 / 2} q, \quad t_{r-1}=t_{0}^{1 / 2} q p^{-1 / 2}, \quad t_{r}=-t_{0}^{1 / 2} q p^{1 / 2} .
$$

В результате совершенно уравновешенные тета-гипергеометрические ряды $r+1 E_{r}$ принимают вид

$$
{ }_{r+1} E_{r}=\sum_{\lambda=0}^{\infty} \frac{\theta\left(t_{0} q^{2 \lambda} ; p\right)}{\theta\left(t_{0} ; p\right)} \prod_{m=0}^{r-4} \frac{\theta\left(t_{m} ; p ; q\right)_{\lambda}}{\theta\left(q t_{0} / t_{m} ; p ; q\right)_{\lambda}}(-q z)^{\lambda} .
$$

При $p \rightarrow 0$ ряды (15) сводятся к совершено уравновешенным базисным гипергеометрическим рядам $r-1 \Phi_{r-2}[4]$ аргумента $-q z$ (обрашаем внимание на сдвиг индекса $r$ на -2$)$.

Ряды типа (15) (в других обозначениях, с другим подсчетом числа параметров и выбором $z=-1$ ) были рассмотрены впервые Тураевым и Френкелем [5]. Их анализ был инспирирован точно решаемыми моделями статистической механики, построенными Дате с сотрудниками [3]. В другом контексте в рамках программы по систематическому пересмотру специальных функций одной переменной как “самоподобных" функций (см., например, обзор [6]) эллиптические гипергеометрические ряды были получены Жедановым и автором как решения специального трехчленного рекуррентного соотношения, описывающего новые семейства биортогональных рациональных функций [7].

Тураев и Френкель [5] доказали следующую формулу суммирования:

$$
\begin{aligned}
& \sum_{\lambda=0}^{N} q^{\lambda} \frac{\theta\left(t_{0} q^{2 \lambda} ; p\right)}{\theta\left(t_{0} ; p\right)} \prod_{r=0}^{5} \frac{\theta\left(t_{r} ; p ; q\right)_{\lambda}}{\theta\left(q t_{0} t_{r}^{-1} ; p ; q\right)_{\lambda}}= \\
& \quad=\frac{\theta\left(q t_{0} ; p ; q\right)_{N} \prod_{1 \leqslant r<s \leqslant 3} \theta\left(q t_{0} / t_{r} t_{s} ; p ; q\right)_{N}}{\theta\left(q t_{0} / t_{1} t_{2} t_{3} ; p ; q\right)_{N} \prod_{r=1}^{3} \theta\left(q t_{0} / t_{r} ; p ; q\right)_{N}}
\end{aligned}
$$

где $\prod_{i=1}^{5} t_{i}=q t_{0}^{2}$ и $t_{4}=q^{-N}, N \in \mathbb{N}$. По классификации, введенной в [1], это соответствует замкнутому выражению для обрываюшегося совершенно уравновешенного сбалансированного тета-гипергеометрического ряда ${ }_{10} E_{9}$ при $z=-1$. 
В случае многих переменных общая форма эллиптических гипергеометрических рядов еше не установлена. В литературе предложено несколько типов таких рядов и явных формул суммирования, обобщающих (16). Эти суммы расширяют богатую совокупность тождеств для рядов гипергеометрического типа, которые были найдены впервые Биденхарном, Лауком и Холманом (см., например, [8]) и впоследствии обобщены на базисные гипергеометрические ряды Милне (см., например, [9], [10]) и Густафосоном (см., например, [11], [12]). Варнааром были предложены [13] первые два примера многократных эллиптических гипергеометрических рядов, для одного из которых он доказал обобшение суммы Тураева-Френкеля. Для второго типа рядов Варнаар предположил аналогичную формулу суммирования. Она была доказана для случая обычных и $q$-гипергеометрических рядов ван Диехеном и автором в работе [14] (некоторые предельные случаи этой суммы были доказаны ранее в [15]). Там же было дано частичное доказательство эллиптической суммы. Она была полностью доказана Розенгреном с помощью другой техники [16].

Третий тип рядов был предложен в [17] вместе с эллиптическим расширением многократной суммы Джексона из работ [10], [11], которая была частично доказана на основе инвариантности относительно группы модулярных преобразований. В настоящей статье мы рассматриваем два новых типа многократных эллиптических рядов, изучаем их свойства, обсуждаем соответствуюшие суммы типа Джексона и на основе модулярности показьваем их частичную справедливость. Независимо Розенгрен также рассмотрел эти суммы, доказал их (совместно с суммой, предложенной в [17]) и исследовал ряд их следствий [18].

Точно так же, как и в одномерном случае, многократные тета-гипергеометрические ряды называются полностью эллиптическими, если функции $h_{\ell}(\boldsymbol{\lambda})$ эллиптичны по $\lambda_{j}$ и по всем свободным параметрам, входящим в аргументы $\theta_{1}$-функций. Одна из основных целей настоящей работы состоит в приведении новых аргументов в пользу справедливости следующей гипотезы.

ГИПОТЕЗА. Любой полностью әллиптический гипергеометрический ряд автоматически является модулярно-инвариантным.

Для ряда с $n=1$ (в общей ситуации) и $C_{n}$-сумм, обсуждавшихся в работах [13], [14], $[16],[17]$, это утверждение было доказано в [1]. Докажем его для самой первой формулы суммирования многократного эллиптического гипергеометрического ряда, найденной Варнааром [13] (эта формула была пропушена в анализе [1]), и для упомянутых выше двух новых рядов.

Варнаар доказал [13] следуюший эллиптический аналог многократной $C_{n}$-суммы Шлоссера [19]:

$$
\begin{aligned}
& \sum_{\lambda_{1}, \ldots, \lambda_{n}}^{N}=0 q^{\sum_{j=1}^{n} j \lambda_{j}} \prod_{1 \leqslant i<j \leqslant n} \frac{\theta\left(t_{i} t_{j}^{-1} q^{\lambda_{i}-\lambda_{j}}, t_{i} t_{j} q^{\lambda_{i}+\lambda_{j}} ; p\right)}{\theta\left(t_{i} t_{j}^{-1}, t_{i} t_{j} ; p\right)} \times \\
& \quad \times \prod_{j=1}^{n} \frac{\theta\left(t_{j}^{2} q^{2 \lambda_{j}} ; p\right) \theta\left(t_{j}^{2}, b t_{j}, c t_{j}, d t_{j}, e t_{j}, q^{-N} ; p ; q\right)_{\lambda_{j}}}{\theta\left(t_{j}^{2} ; p\right) \theta\left(q, t_{j} q / b, t_{j} q / c, t_{j} q / d, t_{j} q / e, t_{j}^{2} q^{N+1} ; p ; q\right)_{\lambda_{j}}}=
\end{aligned}
$$




$$
=\prod_{1 \leqslant i<j \leqslant n} \frac{\theta\left(t_{i} t_{j} q^{N} ; p\right)}{\theta\left(t_{i} t_{j} ; p\right)} \prod_{j=1}^{n} \frac{\theta\left(t_{j}^{2} q, q^{2-j} /(b c), q^{2-j} /(b d), q^{2-j} /(c d) ; p ; q\right)_{N}}{\theta\left(t_{j}^{-1} q^{2-n} /(b c d), t_{j} q / b, t_{j} q / c, t_{j} q / d ; p ; q\right)_{N}}
$$

где параметры удовлетворяют условию балансировки $b c d e=q^{N-n+2}$.

ЗАмЕЧАнИЕ 1. Формулы суммирования, приведенные в [13], [19], содержат один дополнительный параметр $a$, но он может быть устранен переопределением переменных $t_{j}, b, c, d, e$.

Теорема 1. Тета-гипергеометрический ряд, стоящий слева в формуле (17), является полностью әллиптическим и модулярно-инвариантным.

ДокАЗАТЕЛЬСтво. Рассмотрим отношения последовательных коэффициентов в ряде $(17)$ :

$$
\begin{aligned}
h_{\ell}(\boldsymbol{\lambda})= & q^{\ell} \frac{\theta\left(t_{\ell}^{2} q^{2 \lambda_{\ell}+2} ; p\right)}{\theta\left(t_{\ell}^{2} q^{2 \lambda_{\ell}} ; p\right)} \prod_{i=1}^{\ell-1} \frac{\theta\left(t_{i} t_{\ell}^{-1} q^{\lambda_{i}-\lambda_{\ell}-1}, t_{i} t_{\ell} q^{\lambda_{i}+\lambda_{\ell}+1} ; p\right)}{\theta\left(t_{i} t_{\ell}^{-1} q^{\lambda_{i}-\lambda_{\ell}}, t_{i} t_{\ell} q^{\lambda_{i}+\lambda_{\ell}} ; p\right)} \times \\
& \times \prod_{j=\ell+1}^{n} \frac{\theta\left(t_{\ell} t_{j}^{-1} q^{\lambda_{\ell}-\lambda_{j}+1}, t_{\ell} t_{j} q^{\lambda_{\ell}+\lambda_{j}+1} ; p\right)}{\theta\left(t_{\ell} t_{j}^{-1} q^{\lambda_{\ell}-\lambda_{j}}, t_{\ell} t_{j} q^{\lambda_{\ell}+\lambda_{j}} ; p\right)} \times \\
& \times \frac{\theta\left(t_{\ell}^{2} q^{\lambda_{\ell}}, b t_{\ell} q^{\lambda_{\ell}}, c t_{\ell} q^{\lambda_{\ell}}, d t_{\ell} q^{\lambda_{\ell}}, e t_{\ell} q^{\lambda_{\ell}}, q^{-N+\lambda_{\ell}} ; p\right)}{\theta\left(q^{\lambda_{\ell}+1}, t_{\ell} q^{\lambda_{\ell}+1} / b, t_{\ell} q^{\lambda_{\ell}+1} / c, t_{\ell} q^{\lambda_{\ell}+1} / d, t_{\ell} q^{\lambda_{\ell}+1} / e, t_{\ell}^{2} q^{N+1+\lambda_{\ell}} ; p\right)}
\end{aligned}
$$

Докажем сначала, что эта функция эллиптична по $\lambda_{k}$. Для этого достаточно заменить $q^{\lambda_{k}}$ на $p q^{\lambda_{k}}$, применить правило $\theta(p z ; p)=-z^{-1} \theta(z ; p)$ и проверить сокрашение всех дополнительных факторов, появляющихся в результате этого. Для $k<\ell$ или $k>\ell$ ситуация достаточно проста и мы не рассматриваем ее. В случае $k=\ell$ первая дробь из тета-функций в (18) дает множитель $q^{-4}$, следуюшие два произведения дают $q^{2(1-n)}$. Последняя дробь, содержащая параметры $b, \ldots, e$, генерирует произведение $q^{2 N+6}(b c d e)^{-2}$. В итоге полный результируюший множитель равен единице согласно условию балансировки.

Для проверки эллиптичности по параметрам и модулярной инвариантности $h_{\ell}(\boldsymbol{\lambda})$ произведем замену $t_{j}, b, c, d, e \rightarrow q^{g_{j}}, q^{\beta}, q^{\gamma}, q^{\delta}, q^{\epsilon}$. После этого мы можем переписать (18) в терминах эллиптических чисел следующим образом:

$$
\begin{aligned}
h_{\ell}(\boldsymbol{\lambda})= & \frac{\left[2 g_{\ell}+2 \lambda_{\ell}+2\right]}{\left[2 g_{\ell}+2 \lambda_{\ell}\right]} \prod_{\substack{i=1 \\
i \neq \ell}}^{n} \frac{\left[g_{i}-g_{\ell}+\lambda_{i}-\lambda_{\ell}-1, g_{i}+g_{\ell}+\lambda_{i}+\lambda_{\ell}+1\right]}{\left[g_{i}-g_{\ell}+\lambda_{i}-\lambda_{\ell}, g_{i}+g_{\ell}+\lambda_{i}+\lambda_{\ell}\right]} \times \\
& \times \frac{\left[2 g_{\ell}+\lambda_{\ell}, \beta+g_{\ell}+\lambda_{\ell}, \gamma+g_{\ell}+\lambda_{\ell}\right]}{\left[\lambda_{\ell}+1, g_{\ell}+\lambda_{\ell}+1-\beta, g_{\ell}+\lambda_{\ell}+1-\gamma\right]} \times \\
& \times \frac{\left[\delta+g_{\ell}+\lambda_{\ell}, \epsilon+g_{\ell}+\lambda_{\ell}, \lambda_{\ell}-N\right]}{\left[g_{\ell}+\lambda_{\ell}+1-\delta, g_{\ell}+\lambda_{\ell}+1-\epsilon, 2 g_{\ell}+N+1+\lambda_{\ell}\right]} .
\end{aligned}
$$

Из этого представления следует, что при переписывании (17) в терминах эллиптических чисел первый множитель $q^{\sum_{j=1}^{n} j \lambda_{j}}$ исчезает. В итоге одновременная перестановка 
переменных $\lambda_{j}, \lambda_{k}$ и параметров $g_{j}, g_{k}$ не меняет коэффициентов $c(\boldsymbol{\lambda})$ (имеет место одинаковое число изменений знаков в числителе и знаменателе $c(\boldsymbol{\lambda})$, происходящих из-за нечетности $[-u]=-[u])$. Таким образом, инвариантность относительно симметрической группы естественным образом реализована в этих рядах.

Эллиптичность по $g_{k}$ в (19) следует из инвариантности (18) относительно сдвига $t_{k} \rightarrow p t_{k}$. Для $k<\ell$ или $k>\ell$ это легко проверяется. Для $k=\ell$ первая дробь тета-функций порождает множитель $q^{-4}$, следуюшие два произведения дают $q^{2(1-n)}$. Остаток в $h_{\ell}$ приводит к множителю $q^{2 N+6}(b c d e)^{-2}$, и полный множитель равен единице благодаря условию балансировки. Для проверки эллиптичности по $\beta$ (или, что эквивалентно, по $\gamma$ или $\delta$ ) заменим $b \rightarrow p b, e \rightarrow p^{-1} e$ в (18). Только последняя дробь дает некоторые вклады, и, поскольку $\theta\left(p^{-1} z ; p\right)=-p^{-1} z \theta(z ; p)$, они сокрашают друг друга.

Обратимся теперь к модулярной инвариантности. Поскольку числитель и знаменатель $h_{\ell}$ содержит одинаковое число $\theta_{1}$-функций, численные факторы, появляюшиеся из-за преобразований $\tau \rightarrow \tau+1, \tau \rightarrow-1 / \tau$ в $(9),(10)$, сокрашаются. Таким образом, достаточно проверить, что разность квадратов аргументов эллиптических чисел в числителе и знаменателе равна нулю. Для первой дроби тета-функций в (19) эта разность равна $4+8\left(g_{\ell}+\lambda_{\ell}\right)$. Следующее произведение дает $2\left(1+2\left(g_{\ell}+\lambda_{\ell}\right)\right)(n-1)$. Остаток $h_{\ell}$ генерирует разность $2(\beta+\gamma+\delta+\epsilon)\left(1+2\left(g_{\ell}+\lambda_{\ell}\right)\right)-4-4\left(g_{\ell}+\lambda_{\ell}\right)(N+3)-2(N+1)$. Полная сумма этих выражений равна нулю, что доказывает модулярную инвариантность функции $h_{\ell}$. По терминологии Эйхлера-Загира [20] это означает, что все функции $h_{\ell}(\boldsymbol{\lambda}), \ell=1, \ldots, n$, в (19) являются мероморфными формами Якоби веса 0 и индекса 0. Все вместе они образуют некоторую структуру, связанную с системой корней $C_{n}$.

Хотя совпадение левой и правой частей (17) было доказано в работе [13], для полноты мы проверили, что выражение справа в (17) является эллиптической функцией всех параметров $g_{j}, \beta, \gamma, \delta$ и оно модулярно-инвариантно.

Во время работы над эллиптической цепочкой Бэйли автор познакомился со статьей Бхатнагара и Шлоссера [22], в которой изучались различные преобразования Бэйли для многократных рядов $10 \Phi_{9}$. Цепочка Бэйли, построенная в [21], генерирует бесконечную последовательность таких преобразований для одномерных эллиптических гипергеометрических рядов. Сравнение [21] и [22] естественным образом привело к предположению, что многие результаты работы [22] должны допускать эллиптическое обобщение. В частности, это предположение подразумевает справедливость следующих эллиптических аналогов многократных сумм Джексона, найденных Милне [9], Шлоссером [23] и Бхатнагаром [24]:

эллиптическая $A_{n}$-сумма Милне

$$
\begin{aligned}
\sum_{\substack{0 \leqslant \lambda_{j} \leqslant N_{j} \\
j=1, \ldots, n}} q^{\sum_{j=1}^{n} j \lambda_{j}} \prod_{j=1}^{n} \frac{\theta\left(t_{j} q^{\lambda_{j}+|\lambda|} ; p\right)}{\theta\left(t_{j} ; p\right)} \prod_{1 \leqslant i<j \leqslant n} \frac{\theta\left(t_{i} t_{j}^{-1} q^{\lambda_{i}-\lambda_{j}} ; p\right)}{\theta\left(t_{i} t_{j}^{-1} ; p\right)} \times \\
\quad \times \prod_{i, j=1}^{n} \frac{\theta\left(t_{i} t_{j}^{-1} q^{-N_{j}} ; p ; q\right)_{\lambda_{i}}}{\theta\left(q t_{i} t_{j}^{-1} ; p ; q\right)_{\lambda_{i}}} \prod_{j=1}^{n} \frac{\theta\left(t_{j} ; p ; q\right)_{|\lambda|}}{\theta\left(t_{j} q^{1+N_{j}} ; p ; q\right)_{|\lambda|}} \times
\end{aligned}
$$




$$
\begin{gathered}
\times \frac{\theta(b, c ; p ; q)_{|\lambda|}}{\theta(q / d, q / e ; p ; q)_{|\lambda|}} \prod_{j=1}^{n} \frac{\theta\left(d t_{j}, e t_{j} ; p ; q\right)_{\lambda_{j}}}{\theta\left(t_{j} q / b, t_{j} q / c ; p ; q\right)_{\lambda_{j}}}= \\
=\frac{\theta(q /(b d), q /(c d) ; p ; q)_{|N|}}{\theta(q / d, q /(b c d) ; p ; q)_{|N|}} \prod_{j=1}^{n} \frac{\theta\left(t_{j} q, t_{j} q /(b c) ; p ; q\right)_{N_{j}}}{\theta\left(t_{j} q / b, t_{j} q / c ; p ; q\right)_{N_{j}}}
\end{gathered}
$$

где $|\lambda|=\lambda_{1}+\cdots+\lambda_{n},|N|=N_{1}+\cdots+N_{n}$ и $b c d e=q^{1+|N|}$;

эллиптическая $D_{n}$-сумма Бхатнагара-Шлоссера (как заявлено в [22], $D_{n}$-суммы работ [23], [24] сушественно эквивалентны друг другу)

$$
\begin{aligned}
& \sum_{\substack{0 \leqslant \lambda_{j} \leqslant N_{j} \\
j=1, \ldots, n}} q^{\sum_{j=1}^{n} j \lambda_{j}} \prod_{j=1}^{n} \frac{\theta\left(t_{j} q^{\lambda_{j}+|\lambda|} ; p\right)}{\theta\left(t_{j} ; p\right)} \prod_{1 \leqslant i<j \leqslant n} \frac{\theta\left(t_{i} t_{j}^{-1} q^{\lambda_{i}-\lambda_{j}} ; p\right)}{\theta\left(t_{i} t_{j}^{-1} ; p\right)} \times \\
& \quad \times \prod_{1 \leqslant i<j \leqslant n} \theta\left(t_{i} t_{j} q /(b c d) ; p ; q\right)_{\lambda_{i}+\lambda_{j}}^{-1} \prod_{i, j=1}^{n} \theta\left(t_{i} t_{j} q^{1+N_{j}} /(b c d) ; p ; q\right)_{\lambda_{i}} \times \\
& \quad \times \prod_{i, j=1}^{n} \frac{\theta\left(t_{i} t_{j}^{-1} q^{-N_{j}} ; p ; q\right)_{\lambda_{i}}}{\theta\left(q t_{i} t_{j}^{-1} ; p ; q\right)_{\lambda_{i}}} \prod_{j=1}^{n} \frac{\theta\left(t_{j} ; p ; q\right)_{|\lambda|} \theta\left(b c d t_{j}^{-1} ; p ; q\right)_{|\lambda|-\lambda_{j}}}{\theta\left(t_{j} q^{1+N_{j}}, b c d t_{j}^{-1} q^{-N_{j}} ; p ; q\right)_{|\lambda|}} \times \\
& \quad \times \frac{\theta(b, c, d ; p ; q)_{|\lambda|}}{\prod_{j=1}^{n} \theta\left(t_{j} q / b, t_{j} q / c, t_{j} q / d ; p ; q\right)_{\lambda_{j}}}= \\
& =\prod_{j=1}^{n} \frac{\theta\left(t_{j} q, t_{j} q /(b c), t_{j} q /(b d), t_{j} q /(c d) ; p ; q\right)_{N_{j}}}{\theta\left(t_{j} q /(b c d), t_{j} q / b, t_{j} q / c, t_{j} q / d ; p ; q\right)_{N_{j}}} .
\end{aligned}
$$

ЗАмЕчАниЕ 2 . В представлении $A_{n}$-суммы Милне, приведенном в приложении статьи [22], имеется дополнительный параметр $a$, но он может быть устранен переопределением $t_{j}, d$ и $e$. Аналогично в версии Шлоссера $D_{n}$-суммы, приведенной там же, дополнительный параметр $a$ может быть устранен переопределением $t_{j}$ (то же самое справедливо для версии Бхатнагара этой суммы).

Теорема 2. Тета-гипергеометрические $A_{n}-u D_{n}$-ряды, стоящие слева в равенствах (20) и (21), являются полностью әллиптическими и модулярно-инвариантными. Соответствующие выражения в правых частях обладают теми же свойствами.

ДокАЗАТЕльСтво. Рассмотрим функции $h_{\ell}(\boldsymbol{\lambda})$ в $A_{n}$-случае:

$$
\begin{aligned}
h_{\ell}(\boldsymbol{\lambda})= & q^{\ell} \prod_{\substack{j=1 \\
j \neq \ell}}^{n} \frac{\theta\left(t_{j} q^{\lambda_{j}+|\lambda|+1} ; p\right)}{\theta\left(t_{j} q^{\lambda_{j}+|\lambda|} ; p\right)} \frac{\theta\left(t_{\ell} q^{\lambda_{\ell}+|\lambda|+2} ; p\right)}{\theta\left(t_{\ell} q^{\lambda_{\ell}+|\lambda|} ; p\right)} \times \\
& \times \prod_{i=1}^{\ell-1} \frac{\theta\left(t_{i} t_{\ell}^{-1} q^{\lambda_{i}-\lambda_{\ell}-1} ; p\right)}{\theta\left(t_{i} t_{\ell}^{-1} q^{\lambda_{i}-\lambda_{\ell}} ; p\right)} \prod_{j=\ell+1}^{n} \frac{\theta\left(t_{\ell} t_{j}^{-1} q^{\lambda_{\ell}-\lambda_{j}+1} ; p\right)}{\theta\left(t_{\ell} t_{j}^{-1} q^{\lambda_{\ell}-\lambda_{j}} ; p\right)} \times
\end{aligned}
$$




$$
\begin{aligned}
& \times \prod_{j=1}^{n} \frac{\theta\left(t_{\ell} t_{j}^{-1} q^{\lambda_{\ell}-N_{j}}, t_{j} q^{|\lambda|} ; p\right)}{\theta\left(t_{\ell} t_{j}^{-1} q^{\lambda_{\ell}+1}, t_{j} q^{1+N_{j}+|\lambda|} ; p\right)} \times \\
& \times \frac{\theta\left(b q^{|\lambda|}, c q^{|\lambda|}, d t_{\ell} q^{\lambda_{\ell}}, e t_{\ell} q^{\lambda_{\ell}} ; p\right)}{\theta\left(q^{|\lambda|+1} / d, q^{|\lambda|+1} / e, t_{\ell} q^{\lambda_{\ell}+1} / b, t_{\ell} q^{\lambda_{\ell}+1} / c ; p\right)}
\end{aligned}
$$

Докажем сначала эллиптичность $h_{\ell}(\boldsymbol{\lambda})$ по $\lambda_{k}$. Для $k<\ell$ или $k>\ell$ сдвиг $q^{\lambda_{k}} \rightarrow p q^{\lambda_{k}}$ в $h_{\ell}$ приводит к следуюшим дополнительным множителям: $q^{-n}$ и $q^{-2}$ от первых двух дробей тета-функций, соответственно; следующие два произведения дают $q$; из произведения $\prod_{j=1}^{n}$ получается вклад $q^{n+|N|}$, а остаток $h_{\ell}$ генерирует фактор $q^{1-|N|}$. Полный множитель равен единице.

Аналогично, заменяя $q^{\lambda_{\ell}}$ на $p q^{\lambda_{\ell}}$, мы получаем последовательно множители $q^{1-n}$, $q^{-4}, q^{1-\ell}, q^{\ell-n}, q^{2 n+2|N|}$ и $q^{2-2|N|}$, и их перемножение дает единицу. Таким образом, функции $h_{\ell}(\boldsymbol{\lambda})$, порождающие левую часть (20), эллиптичны по всем $\lambda_{k}$.

Для того чтобы увидеть естественное действие симметрической группы, заменим $t_{j}, b$, $c, d, e \rightarrow q^{g_{j}}, q^{\beta}, q^{\gamma}, q^{\delta}, q^{\epsilon}$ и перепишем сумму (20) в терминах эллиптических чисел:

$$
\begin{aligned}
\sum_{\substack{0 \leqslant \lambda_{j} \leqslant N_{j} \\
j=1, \ldots, n}} \prod_{j=1}^{n} \frac{\left[g_{j}+\lambda_{j}+|\lambda|\right]}{\left[g_{j}\right]} \prod_{1 \leqslant i<j \leqslant n} \frac{\left[g_{i}-g_{j}+\lambda_{i}-\lambda_{j}\right]}{\left[g_{i}-g_{j}\right]} \times \\
\quad \times \prod_{i, j=1}^{n} \frac{\left[g_{i}-g_{j}-N_{j}\right]_{\lambda_{i}}}{\left[1+g_{i}-g_{j}\right]_{\lambda_{i}}} \prod_{j=1}^{n} \frac{\left[g_{j}\right]_{|\lambda|}}{\left[g_{j}+1+N_{j}\right]_{|\lambda|}} \times \\
\quad \times \frac{[\beta, \gamma]_{|\lambda|}}{[1-\delta, 1-\epsilon]_{|\lambda|}} \prod_{j=1}^{n} \frac{\left[\delta+g_{j}, \epsilon+g_{j}\right]_{\lambda_{j}}}{\left[g_{j}+1-\beta, g_{j}+1-\gamma\right]_{\lambda_{j}}}= \\
=\frac{[1-\beta-\delta, 1-\gamma-\delta]_{|N|}}{[1-\delta, 1-\beta-\gamma-\delta]_{|N|}} \prod_{j=1}^{n} \frac{\left[1+g_{j}, 1+g_{j}-\beta-\gamma\right]_{N_{j}}}{\left[g_{j}+1-\beta, g_{j}+1-\gamma\right]_{N_{j}}}
\end{aligned}
$$

С делаем перестановку $\left(\lambda_{k}, g_{k}, N_{k}\right)$ и $\left(\lambda_{m}, g_{m}, N_{m}\right)$ для некоторых фиксированных $k$ и $m$. Имеется только два множителя, которые требуют некоторого рассмотрения. Первый, заданный произведением $\prod_{1 \leqslant i<j \leqslant n}$, оказывается симметричным из-за равного числа изменений знака в числителе и знаменателе (точно так же, как это было для суммы (17)). Произведение $\prod_{i, j=1}^{n}$ оказывается симметричным, поскольку оно учитывает все возможные значения обоих индексов $i$ и $j$. Таким образом, согласно определению, данному в начале статьи, мы имеем дело с частным случаем эллиптических гипергеометрических рядов.

Докажем теперь полную эллиптичность функций $h_{\ell}$. Эллиптичность по $\beta$ (или $\gamma$ ), или инвариантность (22) при сдвигах $b \rightarrow p b, e \rightarrow p^{-1} e$, следует из сокращения множителей $-b^{-1} q^{-|\lambda|},-p^{-1} e t_{\ell} q^{\lambda_{\ell}}$ и $-q^{|\lambda|+1} e^{-1},-p b t_{\ell}^{-1} q^{-\lambda_{\ell}-1}$, порожденных числителем и знаменателем последней дроби, соответственно. Аналогичная ситуация имеет место для сдвигов $d \rightarrow p d, e \rightarrow p^{-1} e$, что доказывает эллиптичность по $\delta$.

Существенно более сложное вычисление необходимо для проверки модулярной инвариантности $c(\boldsymbol{\lambda})$ в $(23)$. Рассмотрим разность квадратов аргументов эллиптических 
чисел в числителе и знаменателе $h_{\ell}$. Первая дробь тета-функций дает $n-1+2(|g|-$ $\left.g_{\ell}+n|\lambda|-\lambda_{\ell}\right)$, где $|g|=g_{1}+\cdots+g_{n}$. Вторая дробь дает $4\left(1+|\lambda|+\lambda_{\ell}+g_{\ell}\right)$. Следуюшие два произведения генерируют разность $n-1+2 n\left(g_{\ell}+\lambda_{\ell}\right)-2|g|-2|\lambda|$. Произведение $\prod_{j=1}^{n}$ отношений тета-функций дает вклад $-2(n+|N|)\left(1+g_{\ell}+\lambda_{\ell}+|\lambda|\right)$. Остаток $h_{\ell}$ дает

$$
\begin{gathered}
(\beta+|\lambda|)^{2}+(\gamma+|\lambda|)^{2}+\left(\delta+g_{\ell}+\lambda_{\ell}\right)^{2}+\left(\epsilon+g_{\ell}+\lambda_{\ell}\right)^{2}-(|\lambda|+1-\delta)^{2}- \\
-(|\lambda|+1-\epsilon)^{2}-\left(g_{\ell}+\lambda_{\ell}+1-\beta\right)^{2}-\left(g_{\ell}+\lambda_{\ell}+1-\gamma\right)^{2} .
\end{gathered}
$$

Сумма всех этих громоздких выражений равна нулю, т.е. $h_{\ell}$ инвариантна относительно преобразований (9), (10), порождаюших полную модулярную группу.

Полная эллиптичность и модулярность выражения, стоящего справа в равенстве (23), легко проверяются. Действительно, инвариантность правой части (20) при сдвигах $t_{j} \rightarrow p t_{j}$ или $d \rightarrow p d$ (т.е. эллиптичность по $g_{j}$ или $\delta$ ) очевидна. Сдвиг $b \rightarrow p b$ генерирует фактор $c^{|N|}$ из эллиптических сдвинутых факториалов $\theta(\cdot)_{|N|}$, и он сокрашается дополнительными множителями, обусловленными эллиптическими сдвинутыми факториалами $\theta(\cdot)_{N_{j}}$. По соображениям симметрии это означает эллиптичность и по $\beta$, и по $\gamma$.

Что касается разности квадратов аргументов эллиптических чисел в числителе и знаменателе правой части (23), отношение эллиптических с двинутых факториалов [· $]_{\mid N}$ дает (после упрощений) $-2|N| \beta \gamma$. Произведение отношений $[\cdot]_{N_{j}}$ дает вклад

$$
\sum_{j=1}^{n} N_{j}\left(\left(1+g_{j}\right)^{2}+\left(1+g_{j}-\beta-\gamma\right)^{2}-\left(1+g_{j}-\beta\right)^{2}-\left(1+g_{j}-\gamma\right)^{2}\right)
$$

который сокрашает предыдущее выражение. Это доказывает модулярную инвариантность правой части равенства (23).

Обратимся теперь к $D_{n}$-сумме $(21)$. Отношения коэффишиентов ряда в левой части имеют следующий вид:

$$
\begin{aligned}
h_{\ell}(\boldsymbol{\lambda})= & q^{\ell} \prod_{\substack{j=1 \\
j \neq \ell}}^{n} \frac{\theta\left(t_{j} q^{\lambda_{j}+|\lambda|+1}, b c d t_{j}^{-1} q^{|\lambda|-\lambda_{j}} ; p\right)}{\theta\left(t_{j} q^{\lambda_{j}+|\lambda|}, t_{\ell} t_{j} q^{\lambda_{j}+\lambda_{\ell}+1} /(b c d) ; p\right)} \frac{\theta\left(t_{\ell} q^{\lambda_{\ell}+|\lambda|+2} ; p\right)}{\theta\left(t_{\ell} q^{\lambda_{\ell}+|\lambda|} ; p\right)} \times \\
& \times \prod_{i=1}^{\ell-1} \frac{\theta\left(t_{i} t_{\ell}^{-1} q^{\lambda_{i}-\lambda_{\ell}-1} ; p\right)}{\theta\left(t_{i} t_{\ell}^{-1} q^{\lambda_{i}-\lambda_{\ell}} ; p\right)} \prod_{j=\ell+1}^{n} \frac{\theta\left(t_{\ell} t_{j}^{-1} q^{\lambda_{\ell}-\lambda_{j}+1} ; p\right)}{\theta\left(t_{\ell} t_{j}^{-1} q^{\lambda_{\ell}-\lambda_{j}} ; p\right)} \times \\
& \times \prod_{j=1}^{n} \frac{\theta\left(t_{\ell} t_{j} q^{1+N_{j}+\lambda_{\ell}} /(b c d), t_{\ell} t_{j}^{-1} q^{-N_{j}+\lambda_{\ell}}, t_{j} q^{|\lambda|} ; p\right)}{\theta\left(t_{\ell} t_{j}^{-1} q^{\lambda_{\ell}+1}, t_{j} q^{1+N_{j}+|\lambda|}, b c d t_{j}^{-1} q^{|\lambda|-N_{j}} ; p\right)} \times \\
& \times \frac{\theta\left(b q^{|\lambda|}, c q^{|\lambda|}, d q^{|\lambda|} ; p\right)}{\theta\left(t_{\ell} q^{\lambda_{\ell}+1} / b, t_{\ell} q^{\lambda_{\ell}+1} / c, t_{\ell} q^{\lambda_{\ell}+1} / d ; p\right)} .
\end{aligned}
$$


Сдвиги $q^{\lambda_{k}} \rightarrow p q^{\lambda_{k}}$ не меняют этих функций, т.е. они эллиптичны по переменным $\lambda_{k}$. Для $k<\ell$ или $k>\ell$ это следует из сокрашения множителей квазипериодичности

$$
q^{-n}, \quad-\frac{t_{k} t_{\ell}}{b c d} q^{1+\lambda_{k}+\lambda_{\ell}}, \quad \prod_{\substack{j=1 \\ j \neq k, \ell}}^{n}\left(-\frac{t_{j}}{b c d} q^{\lambda_{j}-|\lambda|}\right)
$$

появляюшихся из произведения $\prod_{j=1, j \neq \ell}^{n}$, фактора $q^{-2}$ (из следуюшего отношения тета-функций), $q$ (из двух следующих произведений) и

$$
\prod_{j=1}^{n}\left(-b c d t_{j}^{-1} q^{|\lambda|+1}\right), \quad-q^{-3|\lambda|} /(b c d)
$$

(из двух последних дробей, соответственно). Для $k=\ell$ мы имеем множители $q^{1-n}$ (из первой дроби),

$$
\prod_{j=1, j \neq \ell}^{n}\left(t_{j}^{2} t_{\ell} q^{2 \lambda_{j}+\lambda_{\ell}-|\lambda|+1} /\left(b^{2} c^{2} d^{2}\right)\right)
$$

(из второй дроби),$q^{-4}$ (из третьей дроби),$q^{1-n}$ (из следуюших двух произведений) и

$$
\prod_{j=1}^{n} \frac{b^{2} c^{2} d^{2}}{t_{\ell} t_{j}^{2}} q^{1+|\lambda|-\lambda_{\ell}}, \quad \frac{t_{\ell}^{3}}{b^{2} c^{2} d^{2}} q^{3\left(1+\lambda_{\ell}-|\lambda|\right)}
$$

(из последних двух строк, соответственно). Их произведение равно единице, т.е. функции $h_{\ell}$ эллиптичны по всем $\lambda_{k}$.

После переписывания суммы (21) в терминах эллиптических чисел,

$$
\begin{aligned}
\sum_{\substack{0 \leqslant \lambda_{j} \leqslant N_{j} \\
j=1, \ldots, n}} & \prod_{j=1}^{n} \frac{\left[g_{j}+\lambda_{j}+|\lambda|\right]}{\left[g_{j}\right]} \prod_{1 \leqslant i<j \leqslant n} \frac{\left[g_{i}-g_{j}+\lambda_{i}-\lambda_{j}\right]}{\left[g_{i}-g_{j}\right]} \times \\
& \times \frac{\prod_{i, j=1}^{n}\left[g_{i}+g_{j}+1+N_{j}-\beta-\gamma-\delta\right]_{\lambda_{i}}}{\prod_{1 \leqslant i<j \leqslant n}\left[g_{i}+g_{j}+1-\beta-\gamma-\delta\right]_{\lambda_{i}+\lambda_{j}}} \times \\
& \times \prod_{i, j=1}^{n} \frac{\left[g_{i}-g_{j}-N_{j}\right]_{\lambda_{i}}}{\left[1+g_{i}-g_{j}\right]_{\lambda_{i}}} \prod_{j=1}^{n} \frac{\left[g_{j}\right]_{|\lambda|}\left[\beta+\gamma+\delta-g_{j}\right]_{|\lambda|-\lambda_{j}}}{\left[g_{j}+1+N_{j}, \beta+\gamma+\delta-g_{j}-N_{j}\right]_{|\lambda|}} \times \\
& \times \frac{\left[\beta, \gamma \mid g_{j}+1-\gamma, g_{j}+1-\delta\right]_{\lambda_{j}}}{\prod_{j=1}^{n}\left[g_{j}+1-\beta, \gamma, g_{j}+1-\gamma-\delta\right]_{N_{j}}} \\
= & \prod_{j=1}^{n} \frac{\left[g_{j}+1, g_{j}+1-\beta-\gamma, g_{j}+1-\beta-\delta, g_{j}+1-\delta\right]_{N_{j}}}{\left[g_{j}+1-\beta-\gamma-\delta, g_{j}+1-\beta, g_{j}+1-\gamma, g_{j}+1-\gamma\right.}
\end{aligned}
$$

перестановочная симметрия между $\left(\lambda_{k}, g_{k}, N_{k}\right)$ и $\left(\lambda_{m}, g_{m}, N_{m}\right)$ для фиксированных $k$ и $m$ становится очевидной. 
Эллиптичность по переменным $g_{k}, k \neq \ell$, следует из сокрашения следующей последовательности множителей в $(24)$, появляюшихся после сдвига $t_{k} \rightarrow p t_{k}$ :

$$
q^{-1}, \quad-t_{k} t_{\ell} q^{1+\lambda_{k}+\lambda_{\ell}} /(b c d), \quad-p^{-1} b c d q^{|\lambda|-\lambda_{k}} t_{k}^{-1}, \quad q, \quad p q^{-1-|\lambda|-\lambda_{\ell}} t_{\ell}^{-1} .
$$

Аналогично для $g_{\ell}$ мы получаем множители (в порядке появления)

$$
\begin{gathered}
\prod_{\substack{j=1 \\
j \neq \ell}}^{n}\left(-\frac{t_{j} t_{\ell} q^{1+\lambda_{j}+\lambda_{\ell}}}{b c d}\right), \quad q^{-2}, \quad q^{1-n}, \quad \prod_{\substack{j=1 \\
j \neq \ell}}^{n}\left(-\frac{b c d q^{-\lambda_{\ell}}}{t_{j} t_{\ell}}\right) \\
-\frac{b c d q^{-1-|\lambda|-2 \lambda_{\ell}}}{t_{\ell}^{3}}, \quad-\frac{t_{\ell}^{3} q^{3+3 \lambda_{\ell}}}{b c d},
\end{gathered}
$$

произведение которых равно единице. Эллиптичность по $\beta$ (или $\gamma, \delta$ ) подтверждается аналогичным вычислением, которое мы опускаем для краткости.

Доказательство модулярности $h_{\ell}$ требует существенно более трудоемкого расчета. Опустим начальное выражение для разности квадратов аргументов эллиптических чисел в $h_{\ell}$. Первая цепочка упрошений в нем приводит к сумме

$$
\begin{aligned}
& 2\left(1+|\lambda|+g_{\ell}+\lambda_{\ell}\right)\left(\lambda_{\ell}-|\lambda|-N_{\ell}+1\right)+\left(2 g_{\ell}-\beta-\gamma-\delta+N_{\ell}+1+\lambda_{\ell}\right)^{2}- \\
& -\left(g_{\ell}-\beta-\gamma-\delta+N_{\ell}-|\lambda|\right)^{2}+\sum_{\rho=\beta, \gamma, \delta}\left((\rho+|\lambda|)^{2}-\left(g_{\ell}+\lambda_{\ell}+1-\rho\right)^{2}\right),
\end{aligned}
$$

которая равна нулю.

Работа с правой частью (21) существенно проще. Ее эллиптичность по параметрам следует из того, что отношение

$$
\frac{\left[g_{j}, g_{j}-\beta-\gamma, g_{j}-\beta-\delta, g_{j}-\gamma-\delta\right]}{\left[g_{j}-\beta-\gamma-\delta, g_{j}-\beta, g_{j}-\gamma, g_{j}-\delta\right]}
$$

эллиптично по всем переменным. Модулярная инвариантность гарантирована следующим равенством:

$g_{j}^{2}+\left(g_{j}-\beta-\gamma\right)^{2}+\left(g_{j}-\beta-\delta\right)^{2}+\left(g_{j}-\gamma-\delta\right)^{2}=\left(g_{j}-\beta-\gamma-\delta\right)^{2}+\left(g_{j}-\beta\right)^{2}+\left(g_{j}-\gamma\right)^{2}+\left(g_{j}-\delta\right)^{2}$.

Таким образом, мы доказали полную эллиптичность и модулярность обеих сторон обеих $A_{n}$ - и $D_{n}$-сумм.

СледствиЕ. $C_{n}$-сумма Варнаара, әллиптическая $A_{n}$-сумма Милне и әллиптическая $D_{n}$-сумма Бхатнагара-ШШлоссера, заданные формулами (17), (20) и (21), соответственно, справедливы при разложсении по малому параметру $\sigma$ до степени $\sigma^{12}$. 
ДокАЗАТЕЛЬСТво. Для произвольно малого $\epsilon>0$ можно подобрать достаточно малое абсолютное значение параметра $\sigma$ такое, что обе стороны равенств (17), (20), (21) будут голоморфны по модулярному параметру $\tau$ в области $\operatorname{Im} \tau>\epsilon$. Являясь $\operatorname{PSL}(2, \mathbb{Z})$-инвариантными функциями, они имеют $\sigma$-разложения вида $\sum_{k=0}^{\infty} m_{k}(\tau) \sigma^{2 k}$, где $m_{k}(\tau)$ суть модулярные формы веса $2 k$. Для $\operatorname{Im} \tau \rightarrow \infty$ (т.е. $p \rightarrow 0$ ) разности выражений левых и правых частей этих равенств обрашаются в нуль. Поэтому в разложении этих разностей по $\sigma$ могут появиться только параболические формы. Поскольку не сушествует параболических модулярных форм с весом меньше 12 , рассматриваемые $C_{n^{-}}$, $A_{n^{-}}, D_{n}$-формулы суммирования справедливы в $\sigma$-разложении до членов степени $\sigma^{12}$. Приведенные аргументы не новы, они уже использовались в работах [14], [17] в аналогичной ситуации.

Результаты данной работы значительно усиливают аргументы в пользу гипотезы, приведенной в начале статьи. Кроме того, они показывают, что свойства полной эллиптичности и модулярной инвариантности дают простой способ построения эллиптических обобшений некоторых тождеств для базисных гипергеометрических рядов. Полные доказательства $C_{n}$-суммы, предложенной в [17], и $A_{n^{-}}, D_{n^{-} \text {-сумм }}(20),(21)$, полученные Розенгреном [18], усиливают последнее утверждение. Здесь уместно задаться вопросом о сушествовании аналогичных тождеств для других систем корней.

В заключение кратко обсудим обобшение подхода работы [1] на ряды, используюшие многомерные тета-функции Римана. В случае общих абелевых многообразий можно потребовать, чтобы функции $h_{\ell}(\boldsymbol{\lambda})(2)$ были мероморфными $2 n$-периодическими функциями, определенными на $n$-мерном комплексном торе. Как известно, такие функции однозначно факторизуются в виде отношения некоторых произведений тета-функций рода $n$, так что коэффициенты $c(\boldsymbol{\lambda})$ описываются некоторыми обшими тета-аналогами сдвинутых факториалов. В этом случае перестановочная симметрия $\mathcal{S}_{n}$, вообше говоря, нарушена, а ее наличие подразумевает некоторый самосогласованный выбор функций $h_{\ell}(\boldsymbol{\lambda})$.

В случае алгебраических кривых в качестве $h_{\ell}$ можно взять мероморфные функции на компактной римановой поверхности $S$ произвольного рода $g$. Рассмотрим несколько подробнее ситуацию при $n=1$. Многомерная тета-функция Римана на якобиане определяется матрицей периодов, порожденной нормированным базисом голоморфных дифференциалов $\boldsymbol{\omega}=\left(\omega_{1}, \ldots, \omega_{g}\right)^{1)}$. Обозначим тета-функцию с нечетными характеристиками как $[\mathbf{u}], \mathbf{u} \in \mathbb{C}^{g}$. В частности, мы имеем $[-\mathbf{u}]=-[\mathbf{u}]$ и $\left[\mathbf{u}+\mathbf{e}_{k}\right]=[\mathbf{u}]$, где $\mathbf{e}_{k}$, $k=1, \ldots, g$, определяют базис единичных векторов в $\mathbb{C}^{g}$. Некоторое представление об общей структуре тета-гипергеометрических рядов на $S$ дает следуюший результат.

ТеОРема 3. Введем некоторую последовательность несовпадающих точек на компактной римановой поверхности рода $g: x_{0}, x_{1}, \ldots, x_{N} \in S$. Пусть $\mathbf{u}_{0}-$ произвольный вектор из $\mathbb{C}^{g}$ и параметры $\mathbf{u}_{i}=\int_{P_{i}}^{Q_{i}} \boldsymbol{\omega}, i=1,2$, для некоторых $P_{i}, Q_{i} \in S$. Тогда имеет место следующая фор.мула суммирования для специального ряда ги-

\footnotetext{
1) Подробнее о теории римановых поверхностей и связанных с ними структур см., например, [25].
} 
пергеометрического типа, определенного на поверхности $S$ :

$$
\begin{gathered}
\sum_{n=0}^{N} \frac{\left[\mathbf{u}_{0}+2 \int_{x_{0}}^{x_{n}}\right]}{\left[\mathbf{u}_{0}\right]} \prod_{j=0}^{n-1} \frac{\left[\mathbf{u}_{0}+\int_{x_{0}}^{x_{j}}, \mathbf{u}_{0}-\mathbf{u}_{1}-\mathbf{u}_{2}+\int_{x_{0}}^{x_{j}}, \mathbf{u}_{1}+\int_{x_{0}}^{x_{j}}, \mathbf{u}_{2}+\int_{x_{0}}^{x_{j}}\right]}{\left[\int_{x_{0}}^{x_{j+1}}, \mathbf{u}_{1}+\mathbf{u}_{2}+\int_{x_{0}}^{x_{j+1}}, \mathbf{u}_{0}-\mathbf{u}_{1}+\int_{x_{0}}^{x_{j+1}}, \mathbf{u}_{0}-\mathbf{u}_{2}+\int_{x_{0}}^{x_{j+1}}\right]}= \\
=\prod_{j=1}^{N} \frac{\left[\mathbf{u}_{0}+\int_{x_{0}}^{x_{j}}, \mathbf{u}_{0}-\mathbf{u}_{1}-\mathbf{u}_{2}+\int_{x_{0}}^{x_{j}}, \mathbf{u}_{1}+\int_{x_{0}}^{x_{j}}, \mathbf{u}_{2}+\int_{x_{0}}^{x_{j}}\right]}{\left[\int_{x_{0}}^{x_{j}}, \mathbf{u}_{1}+\mathbf{u}_{2}+\int_{x_{0}}^{x_{j}}, \mathbf{u}_{0}-\mathbf{u}_{1}+\int_{x_{0}}^{x_{j}}, \mathbf{u}_{0}-\mathbf{u}_{2}+\int_{x_{0}}^{x_{j}}\right]}
\end{gathered}
$$

где $\int_{a}^{b} \equiv \int_{a}^{b} \boldsymbol{\omega}$ и произведения $\prod_{j=0}^{n-1}, \prod_{j=1}^{n}$ при $n=0$ принимаются равньлми единиче.

ДокАЗАТЕЛЬСтво. Для вывода этой формулы нам потребуется тождество Фэя для тета-функций на алгебраических кривых [25]

$$
\begin{aligned}
{[\mathbf{u}} & \left.+\int_{a}^{c} \boldsymbol{\omega}, \mathbf{u}+\int_{b}^{d} \boldsymbol{\omega}, \int_{c}^{b} \boldsymbol{\omega}, \int_{a}^{d} \boldsymbol{\omega}\right]+\left[\mathbf{u}+\int_{b}^{c} \boldsymbol{\omega}, \mathbf{u}+\int_{a}^{d} \boldsymbol{\omega}, \int_{a}^{c} \boldsymbol{\omega}, \int_{b}^{d} \boldsymbol{\omega}\right]= \\
& =\left[\mathbf{u}, \mathbf{u}+\int_{a}^{c} \boldsymbol{\omega}+\int_{b}^{d} \boldsymbol{\omega}, \int_{c}^{d} \boldsymbol{\omega}, \int_{a}^{b} \boldsymbol{\omega}\right]
\end{aligned}
$$

справедливое при произвольных $\mathbf{u} \in \mathbb{C}^{g}$ и $a, b, c, d \in S$. Отметим также, что $\int_{a}^{c} \boldsymbol{\omega}+$ $\int_{b}^{d} \boldsymbol{\omega}=\int_{b}^{c} \boldsymbol{\omega}+\int_{a}^{d} \boldsymbol{\omega}$.

Запишем равенство (26) как $\sum_{n=0}^{N} c_{n}=s_{N}, c_{0}=s_{0}=1$. Покажем, что $c_{n}=s_{n}-$ $s_{n-1}$, где $s_{-1}=0$. Действительно,

$$
1-\frac{s_{n-1}}{s_{n}}=1-\frac{\left[\int_{x_{0}}^{x_{n}}, \mathbf{u}_{1}+\mathbf{u}_{2}+\int_{x_{0}}^{x_{n}}, \mathbf{u}_{0}-\mathbf{u}_{1}+\int_{x_{0}}^{x_{n}}, \mathbf{u}_{0}-\mathbf{u}_{2}+\int_{x_{0}}^{x_{n}}\right]}{\left[\mathbf{u}_{0}+\int_{x_{0}}^{x_{n}}, \mathbf{u}_{0}-\mathbf{u}_{1}-\mathbf{u}_{2}+\int_{x_{0}}^{x_{n}}, \mathbf{u}_{1}+\int_{x_{0}}^{x_{n}}, \mathbf{u}_{2}+\int_{x_{0}}^{x_{n}}\right]} .
$$

Подставляя параметры

$$
\mathbf{u} \equiv \mathbf{u}_{0}-\mathbf{u}_{2}+\int_{x_{0}}^{x_{n}} \omega, \quad \int_{c}^{d} \boldsymbol{\omega} \equiv \int_{x_{0}}^{x_{n}} \boldsymbol{\omega}, \quad \int_{d}^{b} \boldsymbol{\omega} \equiv \mathbf{u}_{1}, \quad \int_{a}^{c} \boldsymbol{\omega} \equiv \mathbf{u}_{2}
$$

в тождество Фэя (27), получаем

$$
1-\frac{s_{n-1}}{s_{n}}=\frac{\left[\mathbf{u}_{1}, \mathbf{u}_{2}, \mathbf{u}_{0}-\mathbf{u}_{1}-\mathbf{u}_{2}, \mathbf{u}_{0}+2 \int_{x_{0}}^{x_{n}}\right]}{\left[\mathbf{u}_{0}+\int_{x_{0}}^{x_{n}}, \mathbf{u}_{0}-\mathbf{u}_{1}-\mathbf{u}_{2}+\int_{x_{0}}^{x_{n}}, \mathbf{u}_{1}+\int_{x_{0}}^{x_{n}}, \mathbf{u}_{2}+\int_{x_{0}}^{x_{n}}\right]} .
$$

Легко видеть, что произведение этого выражения на $s_{n}$ дает $c_{n}$. Очевидно, что $\sum_{n=0}^{N}\left(s_{n}-s_{n-1}\right)=s_{N}$, и это доказывает формулу (26).

В случае эллиптических кривых (т.е. для $g=1$ ) при выборе $\omega=d x, x_{j}=\sigma j$ формула (26) представляет собой частный случай суммы Тураева-Френкеля (16), получающийся специальным выбором одного из параметров (см. также формулу (4.1) в [21]). 
Отношения $c_{n+1} / c_{n}$ для ряда в левой части равенства $(26)$ имеют вид

$$
h(n)=\frac{\left[\mathbf{u}_{0}+2 \int_{x_{0}}^{x_{n+1}}, \mathbf{u}_{0}+\int_{x_{0}}^{x_{n}}, \mathbf{u}_{0}-\mathbf{u}_{1}-\mathbf{u}_{2}+\int_{x_{0}}^{x_{n}}, \mathbf{u}_{1}+\int_{x_{0}}^{x_{n}}, \mathbf{u}_{2}+\int_{x_{0}}^{x_{n}}\right]}{\left[\mathbf{u}_{0}+2 \int_{x_{0}}^{x_{n}}, \int_{x_{0}}^{x_{n+1}}, \mathbf{u}_{1}+\mathbf{u}_{2}+\int_{x_{0}}^{x_{n+1}}, \mathbf{u}_{0}-\mathbf{u}_{1}+\int_{x_{0}}^{x_{n+1}}, \mathbf{u}_{0}-\mathbf{u}_{2}+\int_{x_{0}}^{x_{n+1}}\right]} .
$$

Если рассматривать $n$ как точку на комплексной плоскости и отождествить ее с переменной, униформизуюшей риманову поверхность $S, x_{n}=x(n)$, то полученная функция $h(n)$ является мероморфной функцией $n$, как это было в эллиптическом случае. Такое отождествление инспирирует следующее определение гамма-функции для компактных римановых поверхностей рода $g$ :

$$
\Gamma^{(g)}(w+1)=\left[\mathbf{u}+\int_{a}^{x(w)} \boldsymbol{\omega}\right] \Gamma^{(g)}(w),
$$

где $\mathbf{u} \in \mathbb{C}^{g}$ и $a \in S$ произвольны. При $g=1$ это определение ведет к эллиптической гамма-функции, рассмотренной еще Джексоном (см. краткое обсуждение в приложении [21] и соответствующие ссылки). В принципе можно записать $\Gamma^{(g)}(w)$ в виде некоторого бесконечного произведения по орбите фуксовой группы, автоморфные функции которой униформизуют $S$, но не ясно, можно ли эффективно работать с таким формальным представлением. Позднее автор надеется рассмотреть более подробно эти и другие проблемы, затрагиваемые в данной статье.

Благодарности. Автор благодарен Г. Е. Андрюсу, А. Берковичу, Я. Ф. ван Диехену за полезные обсуждения возможных следствий эллиптической цепочки Бэйли, а также Х. Розенгрену за подтверждение (до публикации [18]), что он доказал суммы (20), (21). Обсуждения с Д. Загиром и Ю. И. Маниным в апреле 2002 г. возможностей построения тета-гипергеометрических рядов произвольного рода также сыграли стимулирующую роль. Автор благодарен Институту математики им. Макса Планка в Бонне за поддержку и гостеприимство. Работа была частично поддержана грантом РФФИ (№ 00-01-00299).

\section{Список литературы}

[1] V. P. Spiridonov. Theta hypergeometric series. In: Asymptotic Combinatorics with Application to Mathematical Physics (St. Petersburg, July 9-22, 2001). Eds. V. A. Malyshev, A. M. Vershik. Dordrecht: Kluwer, 2002. P. 307-327.

[2] E. T. Whittaker, G. N. Watson. A Course of Modern Analysis. Cambridge: Cambridge Univ. Press, 1986.

[3] E. Date, M. Jimbo, A. Kuniba, T. Miwa, M. Okado. Adv. Stud. Pure Math. 1988. V. 16. P. 17-122.

[4] G. Gasper, M. Rahman. Basic Hypergeometric Series. Encyclopedia of Mathematics and its Applications. V. 35. Cambridge: Cambridge Univ. Press, 1990.

[5] I. B. Frenkel, V. G. Turaev. Elliptic solutions of the Yang-Baxter equation and modular hypergeometric functions. In: The Arnold-Gelfand Mathematical Seminars: Geometry and Singularity Theory. Eds. V. I. Arnold et al. Boston, MA: Birkhäuser, 1997. P. 171-204.

[6] V.P. Spiridonov. The factorization method, self-similar potentials and quantum algebras. In: Special Functions-2000: Current Perspective and Future Directions. Proc. of the NATO Adv. 
Study Institute (Tempe, USA, May 29-June 9, 2000). NATO Sci. Ser. II. Math. Phys. Chem. V. 30. Eds. J. Bustoz, M. E. H. Ismail, S. K. Suslov. Dordrecht: Kluwer, 2001. P. 335-364.

[7] V.P. Spiridonov, A.S. Zhedanov. Commun. Math. Phys. 2000. V. 210. P. 49-83.

[8] W. J. Holman, L. C. Biedenharn, J. D. Louck. SIAM J. Math. Anal. 1976. V. 7. P. 529-541.

[9] S. C. Milne. The multidimensional ${ }_{1} \Psi_{1}$ sum and Macdonald identities for $A_{l}^{(1)}$. In: Theta Functions. Proc. 35th Summer Res. Inst. Bowdoin Coll. on Theta Functions (Brunwick, ME, July 6-24, 1987). Proc. Symp. Pure Math. V. 49. Part. 2. Eds. L. Ehrenpreis, R. C. Gunning. Providence, RI: AMS, 1989. P. 323-359.

[10] S. C. Milne, G. M. Lilly. Discrete Math. 1995. V. 139. P. 319-346.

[11] R. Y. Denis, R. A. Gustafson. SIAM J. Math. Anal. 1992. V. 23. P. 552-561.

[12] R.A. Gustafson. The Macdonald identities for affine root systems of classical type and hypergeometric series very-well-poised on semisimple Lie algebras. In: Proc. of the Ramanujan Birth Century Year International Symposium on Analysis (Pune, December 26-28, 1987). Eds. N. K. Thakare, K. C. Sharma, T. T. Raghunathan. New Dehli: Macmillan Co. of India, 1989. P. $185-224$.

[13] S. O. Warnaar. Constr. Approx. 2002. V. 18. P. 479-502.

[14] J.F. van Diejen, V. P. Spiridonov. Math. Res. Lett. 2000. V. 7. P. 729-746.

[15] J. F. van Diejen. Publ. Res. Inst. Math. Sci. 1997. V. 33. P. 483-508.

[16] H. Rosengren. Contemp. Math. 2001. V. 291. P. 193-202.

[17] J. F. van Diejen, V. P. Spiridonov. Lett. Math. Phys. 2001. V. 58. P. 223-238.

[18] H. Rosengren. Elliptic hypergeometric series on root systems. math.CA/0207046.

[19] M. Schlosser. Discrete Math. 2000. V. 210. P. 151-169.

[20] M. Eichler, D. Zagier. The Theory of Jacobi Forms. Progr. in Math. V. 55. Boston: Birkhäuser, 1985.

[21] V. P. Spiridonov. Int. Math. Res. Notices. 2002. V. 37. P. 1945-1977.

[22] G. Bhatnagar, M. Schlosser. Constr. Approx. 1998. V. 14. P. 531-567.

[23] M. Schlosser. Ramanujan J. 1997. V. 1. P. 243-274.

[24] G. Bhatnagar. Ramanujan J. 1999. V. 3. P. 175-203.

[25] Д. Мамфорд. Лекции о тета-функциях. М.: Мир, 1988. 